\title{
Cholécystectomies Laparoscopiques Pour Cholécystite Aigue Lithiasique Versus Lithiase Vésiculaire Symptomatique
}

\author{
James Didier $L$. \\ Ide $K$. \\ Adama $S$. \\ Hama Y. \\ Abdoulaye MB.
}

Département de Chirurgie et Spécialités Chirurgicales,

Hôpital National de Niamey

\section{Chaibou MS.}

\section{Dady $H$.}

Département d'anesthésie, de Réanimation et des Urgences, Hôpital National de Niamey

\section{Adakal $O$.}

Faculté des Sciences de la Santé de l'Université de Maradi, Niger

Idé $\boldsymbol{G}$.

Département de Chirurgie et Spécialités Chirurgicales, Hôpital National de Niamey

\section{Maarouf MI.}

Maternité Issaka Gazobi, Services des Urgences. Niamey

Sidibé $T$.

Département de Radiologie Hôpital National de Niamey

Harouna $Y$.

Département de Chirurgie et Spécialités Chirurgicales, Hôpital National de Niamey

\section{Abarchi $\boldsymbol{H}$.}

Département de Chirurgie et Spécialités Chirurgicales,

Hôpital National Lamorde, Niamey

\section{Sani $R$.}

Département de Chirurgie et Spécialités Chirurgicales, Hôpital National de Niamey 


\section{Abstract}

Introduction: The aim of this work was to highlight the therapeutic and prognostic difficulties between a laparoscopic cholecystectomy for acute gallstone cholecystitis (CAL) and uncomplicated symptomatic vesicular lithiasis (LVS) as well as the reasons for conversion to laparotomy. Patients and Methods: This was a prospective comparative and analytic study over 18 months. Patients admitted and operated for CAL or LVS in the A Surgery Department of the National Hospital of Niamey (HNN) were included. Results: The study involved 61 patients divided into two groups. Group 1 (30 patients) corresponding to patients operated for CAL, group 2 (31 patients) corresponding to patients operated for LVS. Laparoscopic cholecystectomy accounted for $61 \%$ of all cholecystectomies performed and $1.45 \%$ of surgical activity during the same period. The average age in group 1 was 43.7 years with extremes of 14 and 61 years. In group 2, the average age was 38.9 years with extremes ranging from 12 to 55 years. Women were predominantly represented with $63.3 \%$ and $96.7 \%$ respectively for groups 1 and 2. Patients were overweight in 9 cases for group 1 (30\% of cases) and 12 cases in group $2(38 \%), 7 \%$ of cases $)$. Hepatic colic was the main sign of appeal in all patients in both groups. In group 1; 26 out of 30 cases or $86.7 \%$ of cases had leukocytosis, whereas in group 2, leukocytosis was normal in 30 cases, ie $96.8 \%$ of cases. Accessibility of the vesicle was difficult in $73.3 \%$ of cases in group 1 against $22.6 \%$ of cases in group 2 . The vesicle was distended and necrotic in groups 1 in $76.7 \%$ and 10 respectively. \% of cases. On the other hand, in $25.8 \%$ of cases, the vesicle was distended and without any necrosis in group 2. The rate of conversion to laparotomy was $6.55 \%$ (4 cases) and exclusively concerned group 1. Operative follow-up immediate outcomes were simple in $98.34 \%$ of cases. The complications involved 2 patients in group 1 (1.66\% of the total), including parietal suppuration and biliary leakage. Mean operative time was $68.7 \mathrm{~min}$ in group 1 versus $41.6 \mathrm{~min}$ in group 2. Mean duration of hospitalization was 4.3 days with extremes between 2 and 10 days in group 1 versus 1,7 days with extremes ranging from 1 to 7 days in group 2. Mortality was zero. Conclusion: In recent years, laparoscopic surgery has made remarkable progress in Niger. Laparoscopic cholecystectomy seems to be more difficult to perform with significant morbidity in the case of CAL than LVS. The risk of per and postoperative complications can be estimated from the clinical data (acute cholecystitis or symptomatic vesicular lithiasis) and the surgeon's experience. In a cholecystectomy that lasts more than 2 hours, the cumulative risk of complications is higher. 
Keywords: Cholecystectomy, laparoscopy, difficulties, Niger

\section{Résumé}

Introduction : L'objectif de ce travail était de ressortir les difficultés d'ordre thérapeutique et pronostique entre une cholécystectomie laparoscopique pour cholécystite aigue lithiasique (CAL) et lithiase vésiculaire symptomatique non compliquée (LVS) ainsi que les motifs de conversion en laparotomie. Méthodologie: Il s'agissait d'une étude prospective comparative et analytique sur 18 mois. Etaient inclus les patients admis et opérés pour CAL ou LVS au service de chirurgie A de l'Hôpital National de Niamey (HNN). Résultats: L'étude a concerné 61 patients repartis en deux groupes. Le groupe 1 (30 patients) correspondant aux patients opérés pour CAL, le groupe 2 (31 patients) correspondant aux patients opérés pour LVS. La cholécystectomie laparoscopique représentait $61 \%$ de 1 'ensemble des cholécystectomies réalisées et $1,45 \%$ de l'activité chirurgicale au cours de la même période. L'âge moyen dans le groupe 1 était de 43,7 ans avec des extrêmes de 14 et 61 ans. Dans le groupe 2, l'âge moyen était de 38,9 ans avec des extrêmes allant de 12 à 55 ans. Les femmes étaient majoritairement représentées avec $63,3 \%$ et $96,7 \%$ respectivement pour les groupes 1 et 2 . Les patients avaient un surpoids dans 9 cas pour le groupe 1 (30\% des cas) et 12 cas dans le groupe 2 (38,7\% des cas). La colique hépatique était le principal signe d'appel chez tous les malades des deux (2) groupes. Dans le groupe $1 ; 26$ cas sur 30 soit 86,7\% avaient une hyperleucocytose alors que dans le groupe 2 la leucocytose était normale dans 30 cas soit $96,8 \%$ des cas. L'accessibilité de la vésicule était difficile dans 73,3\% des cas dans le groupe 1 contre $22,6 \%$ près des cas dans le groupe 2 . La vésicule était distendue et nécrotique dans les groupes 1 respectivement dans $76,7 \%$ et $10 \%$ des cas. Par contre dans $25,8 \%$ des cas, la vésicule était distendue et sans aucune nécrose dans le groupe 2. Le taux de conversion en laparotomie était de 6,55\% (4 cas) et a exclusivement concerné le groupe 1. Les suites opératoires immédiates ont été simples dans 98,34\% des cas. Les complications concernaient 2 patients du groupe 1 (1.66\% du total) dont une suppuration pariétale et une fuite biliaire. La durée opératoire moyenne était de 68,7 min dans le groupe 1 contre 41,6 min dans le groupe 2. La durée moyenne d'hospitalisation était de 4,3 jours avec des extrêmes entre 2 et 10 jours dans le groupe 1 contre 1,7 jours avec des extrêmes allant de 1 à 7 jours dans le groupe 2. La mortalité était nulle. Conclusion : Ces dernières années, la coeliochirurgie a fait des progrès remarquables au Niger. La cholécystectomie laparoscopique semblerait être de réalisation plus difficile avec une morbidité non négligeable en cas de CAL qu'en cas de LVS. Le risque de complications per et post-opératoires peut être estimé à partir des données cliniques (cholécystite aigue ou lithiase vésiculaire symptomatique) 
et de l'expérience du chirurgien. Si une cholécystectomie dure plus de 2 heures, le risque cumulatif de complications est plus élevé.

Mots clés : Cholécystectomie, laparoscopie, difficultés, Niger

\section{Introduction}

La cholécystectomie laparoscopique est le gold standard dans le traitement chirurgical de la lithiase vésiculaire (Borie, 2006). Les avantages de cette technique sont connus (Dubois, 1995). Cette technique a révolutionné la chirurgie et un nouvel espace s'ouvrait à la chirurgie digestive et viscérale, espace qui n'a cessé de s'accroitre (Valverde, 2011). L'essor de la cholécystectomie laparoscopique a été international et exponentiel avec le développement de nombreuses techniques telles que la cholécystectomie par trocart unique, les cholécystectomies et la cholangio-pancreatographie rétrograde endoscopique (CPRE) avec cholangiographie per opératoire, la chirurgie robotique... Ces nouvelles avancées élargissent les indications de la laparoscopie dans la prise en charge de la lithiase vésiculaire et ses complications. Les recommandations concluaient que la voie coelioscopique était préférable à la laparotomie pour le traitement des cholécystites aiguës lithiasiques dont les complications sont beaucoup moins importantes par rapport à la voie classique (Gurusamy, 2007). La conversion en laparotomie est une issue de sortie en cas de complication lors de la laparoscopie. L'indication de la colioscopie dans nos pays a été freinée par les difficultés d'acquisition du matériel, de la formation du personnel médical et paramédical à son usage et à sa maintenance (Sani, 2009). Au Niger avec l'ouverture des nouveaux centres de cœlioscopie, la connaissance et les moyens de prise en charge des complications de la cœlioscopie sont impératifs.

\section{Méthodologie}

Il s'agissait d'une étude prospective comparative et analytique réalisée au service de chirurgie viscérale A de l'hôpital National de Niamey sur une période de dix-huit (18) mois allant du 1er juin 2013 au 31 Décembre 2014. Etaient inclus tous les patients admis et opérés par laparoscopie pour cholécystite aigue lithiasique et lithiase vésiculaire simple non compliquée avec ou sans conversion. Ces patients étaient repartis en deux groupes : le groupe 1 correspondant aux patients opérés pour cholécystite aigu lithiasique, le groupe 2 aux patients opérés de lithiase vésiculaire symptomatique. La comparaison entre les deux groupes a porté sur les résultats en termes d'aspects épidémiologiques, des antécédents, d'aspect de la vésicule et de son pédicule, du temps opératoire, des incidents peropératoires, des causes de conversion, et des suites opératoires. N'étaient pas inclus, les patients admis en dehors de la période d'étude, ceux ayant eu une cholécystectomie 
laparoscopique pour cholécystite aiguë non lithiasique et ceux opérés par laparotomie. Le test de $\mathrm{Khi}^{2}$ a été utilisé pour déterminer les différences statistiquement significatives.

\section{Résultats}

Durant la période d'étude, 100 cholécystectomies, tous services confondus, ont été pratiquées sur 4190 interventions en chirurgie viscérale soit 2,38\%. Parmi ces patients, 61 patients ont eu une cholécystectomie par laparoscopie en chirurgie A de l'Hôpital National de Niamey soit $1.45 \%$ des activités chirurgicales et $61 \%$ de l'ensemble des cholécystectomies. Ces patients étaient repartis en deux groupes. Le groupe 1 incluant les patients opérés pour cholécystite aigue lithiasique $(n=30)$ et le groupe 2 ceux opérés de lithiase vésiculaire symptomatique non compliquée $(n=31)$. L'âge moyen dans le groupe 1 était de 43,7(extrêmes : 14 et 61 ans) et dans le groupe 2, il était de 38,9 ans (extrêmes : 12 et 55ans). Il n'y avait pas de différence significative entre les deux groupes $\left(\mathrm{KHI}^{2}=5,1\right.$ et $\left.\mathrm{p}=0,3\right)$. Les femmes étaient majoritairement représentées avec $63,3 \%$ des cas et $96,7 \%$ respectivement pour les groupes 1 et 2 . Le sexe ratio était de 0.58 pour le groupe 1 contre 0,03 pour le groupe 2 . La différence était significative entre le sexe et le groupe d'appartenance des patients $\left(\mathrm{KHI}^{2}=8,8 ; \mathrm{P}=0,003\right)$. Le surpoids est retrouvé chez $30 \%$ des patients dans le groupe 1 contre $38,7 \%$ des patients dans le groupe 2. L'obésité par contre était retrouvée dans $40 \%$ des cas pour le groupe 1 contre $38,7 \%$ dans le groupe 2 . La différence n'était cependant pas significative entre les deux (2) groupes $\left(\mathrm{KHI}^{2}=2,7 ; \mathrm{ddl}=4 ; \mathrm{P}=0,6\right)$.

L'hypercholestérolémie représentait $16,7 \%$ des cas dans le groupe 1 contre 6,5\% dans le groupe 2. Un (1) drépanocytaire était enregistré dans chaque groupe soit $3,30 \%$ pour groupe 1 et $3,20 \%$ pour le groupe 2 . Par ailleurs les antécédents médicaux (HTA,drepanocytose, hyprecholesterolemie) ne variaient pas significativement entre les deux (2) groupes $\left(\mathrm{KHI}^{2}=4 ; \mathrm{ddl}=3 ; \mathrm{P}=0,3\right)$. Deux cas avec antécédent de laparotomie soit $6,7 \%$ sont notifiés dans le groupe 1 tandis qu'aucun cas n'a été signalé dans le groupe 2.27 cas $(90,0 \%)$ étaient référés dans le groupe 1 et 26 cas $(83,9 \%)$ dans le groupe 2 . La différence n'était pas significative entre les deux (2) groupes $\left(\mathrm{KHI}^{2}=5 ; \mathrm{ddl}=2 ; \mathrm{P}=0,08\right)$.

Pour le délai d'admission, l'intervalle de 48 heures à 3 semaines était le plus représenté dans le groupe 1 avec $36,7 \%$ des cas suivi de 3 semaines à plus $33,3 \%$ des cas. De même que dans le groupe 2, les intervalles de 48 heures à 3 semaines et de 3 semaines à plus étaient les plus représentés avec respectivement $48 \%$ et $42 \%$ des cas. Il n'y avait pas de différence statistiquement significative entre les deux (2) groupes $\left(\mathrm{KHI}^{2}=4 ; \mathrm{ddl}=2 ; \mathrm{P}\right.$ $=0,1$ ). 
La colique hépatique était le motif de consultation chez tous les malades des 2 groupes. La présentation clinique ne variait pas significativement entre les deux (2) groupes $\left(\mathrm{KHI}^{2}=5 ; \mathrm{ddl}=2 ; \mathrm{P}=0,06\right)$. Les patients du groupe 1 avaient une fièvre associée à une hyperleucocytose dans 26 cas soit $86,7 \%$ des cas alors que dans le groupe 2 seule une leucocytose isolée a été rapporté dans 1 cas soit 3,2\%. La différence est statistiquement significative entre le taux de globules blancs et le groupe d'appartenance des patients $\left(\mathrm{KHI}^{2}=39,7 \mathrm{ddl}=1 \mathrm{P}=0,000001\right)$.

La bilirubinémie était élevée chez 4 patients du groupe 1 (13,3\%), on retrouve le même taux dans le groupe 2 (4 patients soit 12,9\%). L'échographie était réalisée chez tous les patients du groupe 2 contre $96,7 \%$ des cas dans le groupe 1. La différence n'était pas significative entre les deux (2) groupes $\left(\mathrm{KHI}^{2}=6 ; \mathrm{ddl}=2 ; \mathrm{P}=0,05\right)$.

Sur le plan thérapeutique, l'accessibilité de la vésicule était difficile dans $73,3 \%$ des cas pour le groupe 1 contre $22,6 \%$ des cas pour le groupe 2 (Figure $\mathrm{n}^{\circ} 1$ ). La différence était statistiquement significative entre les deux (2) groupes $\left(\mathrm{KHI}^{2}=13,8 ; \mathrm{ddl}=1 ; \mathrm{P}=0,0002\right)$.

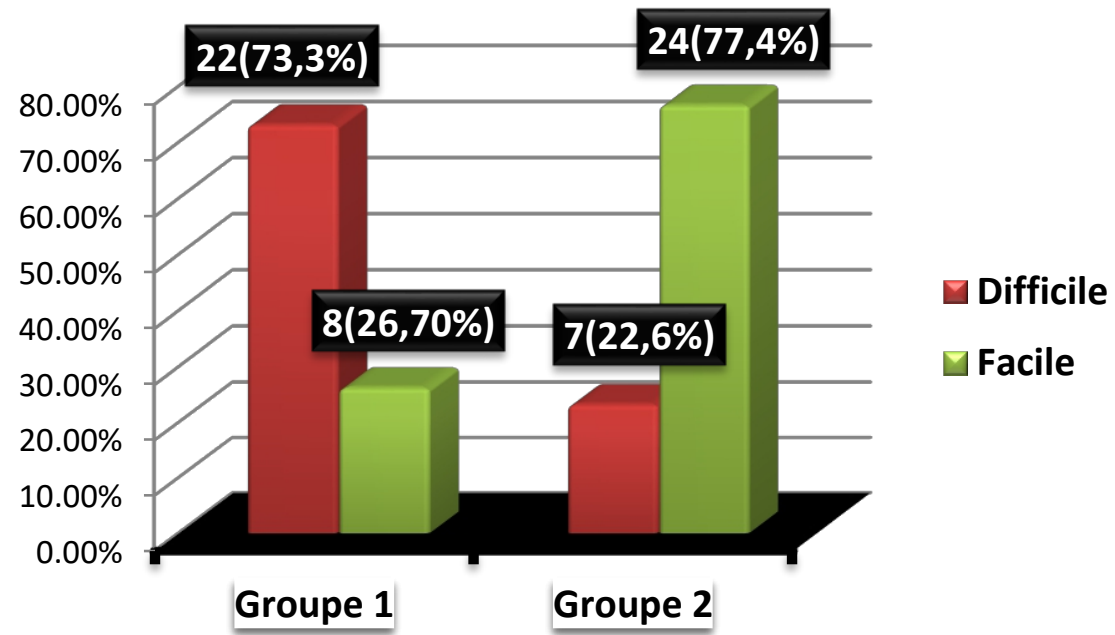

Figure $\mathbf{n}^{0} 1$ : Répartition des patients selon l'accessibilité de la vésicule

La vésicule était distendue chez 23 patients soit 76,7\% des cas du groupe 1 (Tableau I), et dans 3 cas des zones de nécroses. Dans le groupe 2 ; chez 8 patients soit $25,8 \%$ des cas la vésicule était distendue, aucune nécrose vésiculaire n'a été enregistrée dans ce groupe. La différence est significative entre les deux groupes $\left(\mathrm{KHI}^{2}=23,6 \quad \mathrm{ddl}=2 \quad \mathrm{P}=0,000007\right)$.

Tableau I: répartition des patients selon l'aspect de la vésicule

\begin{tabular}{c|cc|c}
\hline \multirow{2}{*}{$\begin{array}{c}\text { Aspect de la } \\
\text { vésicule }\end{array}$} & \multicolumn{2}{|c|}{ Groupe 1 } & \multicolumn{2}{c}{ Groupe 2 } \\
& $\begin{array}{c}\text { Nombre de } \\
\text { cas }\end{array}$ & Pourcentage & Nombre de cas Pourcentage \\
\hline \hline
\end{tabular}




\begin{tabular}{c|cc|cc}
\hline \hline Distendue & $\mathbf{2 3}$ & $\mathbf{7 6 , 7}$ & $\mathbf{8}$ & $\mathbf{2 5 , 8}$ \\
Nécrotique & 3 & 10 & 0 & 0 \\
Normale & 4 & 13,3 & 23 & 74,2 \\
Total & $\mathbf{3 0}$ & $\mathbf{1 0 0}$ & $\mathbf{3 1}$ & $\mathbf{1 0 0}$ \\
\hline \hline
\end{tabular}

Les vésicules et les pédicules cystiques étaient inflammatoires chez 26 patients du groupe 1 (86.7\%) tandis qu'ils étaient libres chez 23 patients du groupe 2 soit 74,2\% des cas. Il y a une relation statistiquement significative entre l'aspect des pédicules et le groupe d'appartenance des patients $(\mathrm{p}=$ 0,0000002 et $\mathrm{KHI}^{2}=22,5 \mathrm{ddl}=1$ ).

Les anomalies artérielles et des voies biliaires étaient exclusivement rencontrées dans le groupe 1 avec un taux de 3,3\% chacune. La différence n'était pas significative entre les deux groupes $\left(\mathrm{KHI}^{2}=2,14 ; \mathrm{ddl}=2 ; \mathrm{P}=0,3\right)$. La conversion avait concerné $13,3 \%$ des cas exclusivement dans le groupe 1 . Les motifs évoqués de conversion sont présentés dans le Tableau I. On note une différence statistiquement significative entre la conversion en laparotomie et le groupe d'appartenance des patients $(\mathrm{p}=0,05)$.

Tableau II : motifs de conversion en laparotomie

\begin{tabular}{llll}
\hline & Age des patients & Diagnostic retenu & \multicolumn{1}{c}{ Cause de conversion } \\
\hline Patiente 1 & 34 ans & CAL & $\begin{array}{l}\text { Adhérences épiplo-pariétales } \\
\text { et digestives }\end{array}$ \\
Patiente 2 & 45 ans & CAL & $\begin{array}{l}\text { Non reconnaissance des } \\
\text { éléments du triangle de } \\
\text { Calot (magma } \\
\text { inflammatoire) }\end{array}$ \\
Patiente 3 & 35 ans & CAL & $\begin{array}{l}\text { Inflammation du pédicule, } \\
\text { rétracté et hémorragique } \\
\text { Vésicule inflammatoire et } \\
\text { saignante avec ouverture de } \\
\text { la vésicule }\end{array}$ \\
Patiente 4 & 43 ans & CAL & \\
& & &
\end{tabular}

Dans le groupe 1, la durée opératoire moyenne était de 68,7 min (extrêmes : 60 à $100 \mathrm{~min}$ );

Par contre, dans le groupe 2, elle est de 41,6 min (extrêmes : 30 à $60 \mathrm{~min}$ ).

Dans le groupe 1, la durée opératoire la plus représentée variait de 60 à 90 min dans $2 / 3$ des cas soit $66,70 \%$. Par contre, dans le groupe 2 la durée opératoire la plus représentée était $\leq 60 \mathrm{~min}$ dans $3 / 4$ des cas soit $74,20 \%$ (Tableau VII). La différence était statistiquement significative entre les deux groupes $\left(\mathrm{KHI}^{2}=19,10 ; \mathrm{ddl}=2 ; \mathrm{P}=0,00007\right)$. Le tableau III ci-dessous illustre la durée opératoire en fonction des deux groupes.

Tableau III. Répartition des patients selon la durée opératoire

\begin{tabular}{|c|c|c|c|c|}
\hline \multirow[t]{2}{*}{$\begin{array}{c}\text { Durée opératoire } \\
\text { (min) }\end{array}$} & \multicolumn{2}{|c|}{ Groupe 1} & \multicolumn{2}{|c|}{ Groupe 2} \\
\hline & Effectif & Pourcentage (\%) & Effectif & Pourcentage (\%) \\
\hline
\end{tabular}




\begin{tabular}{c|cc|cc}
\hline$\leq 60$ & 6 & 20 & 23 & 74,20 \\
60 à 90 & 20 & 66,70 & 8 & 25,80 \\
$>90$ & 4 & 13,30 & 0 & 0 \\
\hline Total & 30 & 100 & 31 & 100 \\
\hline
\end{tabular}

Les suites opératoires ont été simples. Il n'a pas été enregistré d'hémorragies postopératoires. Il y avait eu un cas de fuite biliaire n'ayant pas nécessité de reprise chirurgicale et l'infection pariétale étaient exclusivement observée dans le groupe 1 avec un taux de 3,30\%.

Dans le groupe 1, la durée moyenne d'hospitalisation était de 4,3 jours (extrêmes entre 2 et 10 jours) tandis qu'elle était de 1,7 jour (extrêmes entre 1 et 2 jours) dans le groupe 2 . Dans le groupe 1, la durée d'hospitalisation la plus représentée était $>48$ heures dans $93,3 \%$ des cas tandis que dans le groupe 2 elle était $\leq 48$ heures dans $83,8 \%$ des cas. La différence était statistiquement significative entre la durée d'hospitalisation et le groupe d'appartenance des patients $\left(\mathrm{KHI}^{2}=33,6 ; \mathrm{ddl}=1 ; \mathrm{P}=0,0000001\right)$. Tous les patients des deux (2) groupes étaient sortis guéris.

\section{Discussion}

Sur le plan épidémiologique, la cholécystectomie laparoscopique représente $61 \%$ de l'ensemble des cholécystectomies réalisées et $1,45 \%$ de l'activité chirurgicale. En 2008 cette fréquence est de 53,3\% (Sani, 2009); ceci montre une augmentation de la fréquence de la cholécystectomie laparoscopique par rapport à la cholécystectomie par laparotomie à l'HNN. Diarra, rapporte un taux superposable de 1,2\% de l'activité chirurgicale (Diarra, 2000). Ces résultats sont par contre au-dessus de ceux rapportés par Bonkoungou qui a trouvé un taux 51,6\% de l'ensemble des cholécystectomies (Bonkoungou, 2009). Sanogo $\mathrm{Zz}$ quant a lui a rapporté une fréquence de cholécystectomie laparoscopique de l'ordre de 16,04\% de l'ensemble des activités coelioscopiques (Sanogo Zz, 2006). Dans notre série, l'âge moyen est de 41,22 $\pm 11,27$ avec des extrêmes de 12 et 61 ans. Ces résultats sont proches de ceux rapportés par certaines séries avec un âge moyen autour de 47 ans et des extrêmes de 20 et 80 ans (Bray, 2015 ; Diallo, 2010 ; Fall, 2003). Par contre Sani avait rapporté un âge moyen de 36,4 ans avec des extrêmes de 12 et 72 ans (Sani, 2007). La tranche d'âge comprise entre 30 à 60 ans était la plus représentée dans les 2 groupes. Dans les séries de Bray et Dillo la tranche d'âge respective de 30 à 40 ans (34,2\% des patients) et de 31 à 40 ans $(32,43 \%)$ était la plus concernée (Bray, 2015 ; Diallo, 2010). Globalement Les patients âgés de 40 et plus de 50 ans représentaient $53,3 \%$ dans notre série, ces valeurs sont retrouvées dans la littérature (Hanney, 1999 ; Bennion, 1978 ; Bennion, 1976). L'incidence de la lithiase vésiculaire augmente certes avec l'âge par ailleurs sans inférence particulière sur le pronostic opératoire de la cholécystite aiguë ou la lithiase vésiculaire (Bennion, 1978 ; Grundy, 1985 ; Van der 
Linden, 1970). Le sexe féminin prédomine également dans les 2 groupes. Cette prédominance féminine est retrouvée dans la plupart des séries de 65 à 91\% (Bonkoungou, 2009 ; Bray, 2015). Par contre Fall au Sénégal a retrouvé une prédominance masculine avec 30 patients de sexe masculin, 12 de sexe féminin (Fall, 2003).

Neuf (9) patients (30\%) du groupe 1 et 12 patients $(38,7 \%)$ du groupe 2 ont un surpoids. L'obésité est retrouvée dans $40 \%$ des cas pour le groupe 1 et $38,7 \%$ pour le groupe 2 . Des fréquences plus importantes d'obésité ont été rapportées par Ravelson à Madagascar et Sangaré au Mali avec respectivement $45,18 \%$ et 47,4\% de patients obèses (Ravelson, 2000 ; Sangaré, 2015). En effet il est admis que l'obésité est un facteur de risque de survenue de la lithiase biliaire en général (Trotman, 1982). Un (1) drépanocytaire est enregistré dans chaque groupe. Cette hémoglobinopathie sévit dans la bande subsaharienne et est rapportée par beaucoup de séries africaines (Fall, 2003 ; Sangaré, 2015, Sani, 2009). La drépanocytose est un facteur de risque de lithiase biliaire dont la prévalence africaine est de 4 à 20\% ( Fall, 2003 ).

La colique hépatique est le principal signe d'appel chez tous les malades des deux (2) groupes soit $100 \%$. C'est le mode de manifestation de cette pathologie tel que décrit dans d'autres publications (Sani, 2009; Bonkoungou, 2009).

La majorité des patients de notre série ont été référés d'autres centres. Mais dans la littérature, la plupart des auteurs rapportent les modes d'admissions en urgences surtout dans les cas des cholécystites aigues. Il n y'a pas de différence significative dans le délai d'admission au niveau des 2 groupes dans notre série contrairement à ce qui est rapporté dans la littérature (Sani, 2009 ; Hyun-ki, 2013 ; Brugere, 2003 ; El Madani, 1999)[.

Pour l'hyperleucocytose, la différence entre les deux groupes était significative. Muscari a corrélé le taux élevé de globules blancs avec les dissections difficiles dans la cholécystite aigue (Muscari, 2006). Les mêmes constatations ont été rapportées par Cerdan (Cerdan, 2010). Pour Schäfer les coliques biliaires sont associées à un taux minime de globules blancs contre une hyperleucocytose marquée dans la cholécystite aiguë (Schäfer, 1998)

L'échographie abdominale a constitué le principal moyen diagnostic paraclinique. Ces résultats sont superposables à ceux de Bonkoungou (Bonkoungou, 2009). Le diagnostic de lithiase vésiculaire ou cholécystite aigue était confirmée par l'échographie dans la majorité des cas. Le scanner est rarement obtenu et est demandé en cas de suspicion d'une pancréatite aigüe. Toughrai au Maghreb en 2013 a rapporté 97\% d'échographie et 3\% de scanner (Toughrai, 2013).

L'accès chirurgical de la vésicule a été difficile chez plus de deux tiers de patients dans le groupe de cholécystite aigue contre moins d'un quart dans le groupe de lithiase vésiculaire symptomatique. La différence est 
significative. La difficulté opératoire dans les cholécystites aigues est retrouvée dans les données de la littérature (tableau IV) (Sangaré, 2015 ; Sani, 2009 ; Sabry, 2005 ; Pessaux, 1999). Cette difficulté dans la cholécystite aiguë prédispose aux incidents et complications postopératoires (Sani, 2009 ; Schafer, 1998 ; Sabry, 2005 ; Pessaux, 1999). La différence est statistiquement significative entre l'aspect du pédicule et le groupe d'appartenance des patients (KHI2 $=22,5 ; \mathrm{ddl}=1 ; \mathrm{P}=0,0000002)$. Sangaré a rapporté $21,2 \%$ de vésicules avec inflammation aiguë (Sangaré, 2015). Vincenzo en 2003 a opéré 49 patients pour cholécystite aigue et a retrouvé $42,6 \%$ de cholécystectomies difficiles dont le diagnostic a été posé par l'échographie (Vincenzo, 2003). Pour Vincenzo, la difficulté de la cholécystectomie pour lithiase peut être prédite chez un patient ayant un taux sanguin élevé d'aspartate aminotransférase (Vincenzo, 2003). Dans notre contexte cela peut être lié à un retard de consultation ou un retard de diagnostic. La conséquence de ce retard de prise en charge traduit la complication du tableau clinique. La relation était statistiquement significative entre l'aspect de la vésicule et celui du pédicule et le groupe d'appartenance des patients.

Le taux de conversion dans notre série est de $6,55 \%$ exclusivement dans le groupe 1. La différence était jugée significative entre les deux groupes $(\mathrm{P}=0,05)$. La fuite biliaire et l'infection pariétale étaient exclusivement observées dans le groupe 1. La différence était statistiquement significative entre les deux (2) groupes. J.L Cardin a rapporté 15 plaies vasculaires, 6 plaies viscérales et 69 reprises dans une série de 4000 laparoscopies (Cardin, 2011) ; Bray a rapporté deux (2) complications dont une (1) suppuration pariétale et une (1) hémorragie de paroi avec un taux de 9,1\% (Bray, 2015). K.Bonastos a retrouvé $7,69 \%$ de morbidité sur des cholécystectomies pour lithiase vésiculaire chez les drépanocytaires (Bonastos, 2001). Ces proportions sont inférieures à celles de certains auteurs (Fall, 2003 ; Bray, 2015 ; Leandros, 2000).

La durée opératoire dans notre série est inférieure à celle de Bray qui a trouvé une durée moyenne 90 min avec des extrêmes de 38 et 142 min, dans une série de cholécystites aiguës lithiasiques (Bray, 2015). Elle est également inférieure à celle de Bonkoungou au Burkina qui rapporté une durée moyenne de 117 min avec des extrêmes de 45 et 225 min, dans une série de lithiases vésiculaires symptomatiques (Bonkoungou, 2009). Ces résultats sont par contre proches de ceux de Navez qui trouve une moyenne de 60 min sur des cas de cholécystite aiguë (Navez, 2005). Bonastos rapporte un intervalle moyen de 45 à $90 \mathrm{~min}$, dans une série de lithiases vésiculaires symptomatiques opérées (Bonastos, 2001). Dans la série de Gurusamy, la durée de l'opération a été significativement plus courte dans le groupe précoce par rapport au groupe retardé (Curusamy, 2006). 
Tableau IV: répartitions des taux de conversion, temps opératoire et taux de morbidité selon les auteurs.

\begin{tabular}{|c|c|c|c|}
\hline Auteurs & Taux de conversion & $\begin{array}{l}\text { Temps } \\
\text { opératoire }\end{array}$ & $\begin{array}{l}\text { Taux de } \\
\text { morbidité }\end{array}$ \\
\hline Sani (Sani, 2009) & $4,2 \%$ & & $14,9 \%$ \\
\hline Diop (Diop, 1999) & & $\mathrm{M}=55 \mathrm{mn}$ & \\
\hline Fall (Fall, 2003) & & $\mathrm{M}=33,88 \mathrm{mn}$ & \\
\hline Cardin (Cardin 2011) & $1,70 \%$ & & $0,17 \%$ \\
\hline Sabry (Sabry, 2005) & & $\mathrm{M}=55 \mathrm{mn}$ & \\
\hline Pessaux (Pessaux, 1999) & $22 \%$ & & $13,7 \%$ \\
\hline Schäfer ( Schäfer, 1998) & $0,34 \%$ & & $0,07 \%$ \\
\hline Zehetner ( Zehetner, 2007) & 3 à 9\% & & $1,7 \%$ \\
\hline Johanson ( Johanson, 2003 & 29 à $31 \%$ & 98 à $100 \mathrm{mn}$ & 5 à $8 \%$ \\
\hline Kolla ( Kolla, 2004) & $25 \%$ & 93 à 104 mn & \\
\hline Lai (Lai, 1998) & 21 à $24 \%$ & 106 à $122 \mathrm{mn}$ & \\
\hline Lo $($ Lo, 1998$)$ & 11 à $23 \%$ & 90 à135 mn & \\
\hline Notre étude & $6,55 \%$ & $\mathrm{M}=1 \mathrm{hr}$ & $3,27 \%$ \\
\hline
\end{tabular}

La différence est significative dans la durée d'hospitalisation entre entre les deux groupes (tableau V). Ces résultats sont respectivement proches de ceux de Bray MK qui avait rapporté une moyenne de 3 jours avec des extrêmes allant de 2 à 6 jours, dans une série de cholécystites aiguës lithiasiques et de Bonkoungou qui avait trouvé une durée moyenne d'hospitalisation de 5 jours avec des extrêmes de 2 et 24 jours (Bray, 2015 ; Bounkoungou, 2009 ). Dans la littérature ce délai d'hospitalisation variait de 3 à 7 jours (Bonatsos ,2001).

Tableau V : répartition des périodes d'hospitalisation par auteurs et pays

\begin{tabular}{lll}
\hline Auteurs & Durée d'hospitalisation & Pays \\
\hline Pessaux (Pessaux,1999) & 5 à 6 jr & France \\
Toughrai ( Toughrai,2013) & 2,5 à 5 jrs & Maroc \\
Brugere ( Brugere, 2003) & 5 à 6 jrs & France \\
Gurusamy (Gurusamy, 2006) & 1,25 à 2,31 jrs & France \\
Sockeel ( Sockeel, 2009) & 4,3 à 5,6 jrs & EU \\
Sani (Sani, 2013) & 4 jrs & Niger \\
Johansson (Johansson, 2003) & 2 jrs & EU \\
\hline Notre étude & 1,7 à 4,3 jrs & Niger \\
\hline
\end{tabular}

Aucune mortalité n'avait été observée. Ces résultats sont similaires à ceux de certains auteurs (Bray, 3015; Pessaux, 1999 ; Sani, 2013 ; Marescaux, 1992). Ces résultats sont différents de ceux de certaines séries où la mortalité est rapportée à un taux variant de 1\% à 9\% (Gurusamy, 2006 ; Diop, 1999 ;Ludwig , 2008 ) 


\section{Conclusion}

La cholécystectomie laparoscopique est réalisable dans notre contexte quel que soit le type pathologique. Cette chirurgie mini-invasive permet de traiter les pathologies de la vésicule chez les sujets jeunes et âgés, avec une morbidité faible. Le risque de complications per et post-opératoires peut être estimé à partir des caractéristiques du patient (sexe, âge, score, poids...), des données cliniques (cholécystite aigue ou lithiase vésiculaire symptomatique) et de l'expérience du chirurgien. Si une cholécystectomie dure plus de 2 heures, le risque cumulatif de complications est de 4 fois plus élevé que pour une intervention qui dure entre 30 et 60 minutes. Les complications biliaires surviennent en début d'expérience, diminuant avec l'expérience de l'équipe et la progression de la courbe d'apprentissage. En cas de difficulté opératoire, il faut savoir qu'une conversion peut être plus bénéfique dans ce genre de situations. La cholécystectomie laparoscopique est plus difficile en cas de CAL que dans les cas de LVS.

\section{References:}

1. Borie F et Millat B. Cholécystectomie et exploration de la voie biliaire principale par cœlioscopie. Encycl. Méd. Chir - Appareil digestif. Mise à jour 2006 40-950, 25 p.

2. Dubois F. Berthelot G, Levard H. Coelioscopic cholecystectomy: Experience with 2006 cases. World Journal of Surgery ;1995, 19( 5).pp $748-752$.

3. Valverde A, Mosnier H. Cholécystectomie par laparoscopie. journal de Chirurgie Viscérale ;2011;148( 5) .p 400-407 .

4. Gurusamy KS, Samraj K. Cholecystectomy versus no cholecystectomy in patients with silent gallstones. Cochrane Database Syst Rev ,2007;1.CD006230 -CD006230.

5. Sani R, Garba RM, Harouna YD, et al. La chirurgie laparoscopique dans le service de chirurgie digestive de l'Hôpital National de Niamey. guinée médicale ; 2009 ; vol 63.35-42.

6. Diarra F. Chirurgie de la lithiase biliaire : bilan au service de chirurgie A de l'hôpital point G. Thèse de doctorat en Médecine ; Faculté de Médecine de Pharmacie et D’Odonto-stomatologie ; Bamako, 2000. 386.

7. Bonkoungou G, Sanou A, Kabore F, et al. La Cholécystectomie Laparoscopique Au Burkina Faso : A Propos De 32 Cas Le journal de Coelio-chirurgie ; vol 71 ; 2009 .57-60.

8. Sanogo Zz, Sangare D, Soumare L, et al. La cholecystectomie laparoscopique. Les 30 premiers cas de bamako. Mali Médical 2006 T XXI N 2 15.15-22. 
9. Bray M K, Youssouf M., Seid D K. Cholécystectomie laparoscopique pour cholécystite aiguë lithiasique : à propos de 22 cas colligés à l'hôpital de la renaissance de Ndjamena. The Pan African Medical Journal. 2015; 21:311

10. Diallo AT, Soumaoro LT, Toure A et al. Cholécystite aigue lithiasique : à propos de 73 cas opérés à l'Hôpital National Ignace Deen de Conakry. Revue africaine de Chirurgie et spécialités. 2010 ; 4(7) : 2629.

11. Fall B, Sagna A, Diop PS et al. La cholecystectomie dans la drepanocytose. Annales de chirurgie 2003. Vol 128 ; 702-705.

12. Sani R, Abarchi H, Chaibou MS et al. Cholécystectomie par cœlioscopie : les 100 premiers cas à l'hôpital national de NiameyNiger. J Afr Chir Digest 2007; Vol 7, N¹ : 611 - 617

13. Hanney RM, Carmalt HM, Merret N, Tait N. Use of the Hasson cannula producing major vascular injury at laparoscopy. Surg Endosc $1999 ; 13: 1238-1240$

14. Bennion 1J, Grundy SM. Risk factors for the developpement of cholelithiasis in man. - New Engl. J. Med., 1978, 299, 1161-1167 et 1978, 299, 1221-1227.

15. Bennion 1J, Ginsberg RL, Garnick MB et al. Effects of oral contraceptives on the gallbladder bile of normal women. New Engl. J. Med. 1976 ; 294 : 189-192

16. Grundy SM. Factors affecting biliary lipid composition. In « Gallstones ». - Edited by S. Cohen and R.D. Soloway. - Churchill Livingstone, édit., New York, 1985, 45-71

17. Van Der Linden W, Sunzel H. Early versus delayed operation for acute cholecystitis. Am J Surg 1970; 120 :7-25

18. Ravelson JR, Tovone GX, Ahmad. et al. Résultats de la cholécystectomie cœlioscopique au centre hospitalier de Soavinandriana. J.Med. Ther 2000 ; suppl 2 ; 11-12.

19. Sangaré D, Camara M, Sanogo ZZ, Koumaré S, et al. Cholécystectomie laparoscopique au Mali. État de la pratique à Bamako en 2012 : 340 cas. e-mémoires de l'Académie Nationale de Chirurgie, 2015, 14 (3) : 072-076.

20. Trotman BW, Soloway RD. Pigment gallstone disease: summary of the national institutes of health-international workshop. Hepatology 1982; 2 : 897-884.

21. Sani R, Lassey J D, Mallam Abdou Badé,et al. Laparoscopic cholecystectomy in sickle cell patients in Niger. Panafrican medical journal, 2009 ; (3) ; 1-8.

22. Hyun-Ki Yoon. Acute cholecystitis: We can drain it! Gastrointest Interv 2013; 2:47-49. 
23. Brugère C, Slim K, Lacaine F. Quand et par quelle voie opérer une cholécystite aigue lithiasique ? Annales de chirurgies ; 2003 ; (128). 549-550.

24. El Madani A, Badawy A, Henry C., et al. Cholécystectomie laparoscopique dans les cholécystites aigues. Chirurgie $1999 ; 124$ : 171-6.

25. Muscari F, Dousset B, Sauvanet A. Cholecystectomie difficile. Journal de Chirurgie ; 2006 ; 143(2). p 93-98.

26. Cerdan F, Gabaudan C, Soulier B et al. Complications rares d'une cholécystectomie coelioscopique. Éditions Elsevier Masson, Paris, 2010. J Radiol 2010;91:1265-7.

27. Schafer M, Suter C, Klaiber C, et al. Spilled gallstones after laparoscopic cholecystectomy. A relevant problem? A retrospective analysis of 10,174 laparoscopic cholecystectomies. Surg Endosc1998;12:305-9

28. Toughrai I, Samir A, S AL, et al . Traitement séquentiel de la lithiase biliaire versus chirurgie seule: analyse par le score de propension. Pan African Médical Journal. 2013; 14: 145.

29. Sabry A, Mahmoud W. Khafagy, W et al. Antibiotic Prophylaxis in Elective Laparoscopic Cholécystectomy : A Prospective Study. Egyptian Journal of Surgery; 2005 ; 24( 3).p145-151.

30. Pessaux P, Tuech JJ, Duplessis R, et al. Cholécystectomie célioscopique après 75 ans. Chirurgie $1999 ; 124$ : 419-22

31. Vincenzo N, Antonio A, Giuseppe Di Lauro,et al. Difficult Cholecystectomies:Validity of the Laparoscopic Approach. JSLS ;2003; 7:329-333.

32. Cardin J-L, Johanet H. Le Club Colio. Incidents et accidents peropératoires : suites de 4000 laparoscopies. La série du Club Cœlio. 2011 Elsevier Masson /j.jchirv.2011.04.007

33. Bonatsos G, Birbas K, Toutouzas K, Durakis N. Laparoscopic cholecystectomy in adults with sickle cell disease. Surg Endosc.2001; 15(8): 816-9.

34. Leandros E, Kymionis GD, Konstadoulakis MM et al. .Laparoscopic or open cholecystectomy in patients with sickle cell disease : which approach is superior ? Eur J Surg. 2000; 166 (11): 859-61.

35. Navez B, Gigot J-F. Réparation biliaire primaire pour traumatisme opératoire de la voie biliaire au cours d'une cholécystectomie. Chirurgie des voies biliaires. Masson, paris, 2005. 20-39.

36. Curusamy KS, Samraj K. Early versus delayed laparoscopic cholecystectomy for acute cholecystitis. Cochrane Database Systematic Reviews 2006;4:CD005440. 
37. Diop S, Cisse M, Toure Fall A, et al. News results in clinical severity of homozygous sickle cell anemia in Dakar Senegal. Hematol.Cell Ther., 1999;41: 217-221.

38. Zehetner J, Andreas S, Wolfgang W. Lost gallstones in laparoscopic cholecystectomy: all possible complications. The American Journal of Surgery; 2007; (193). 73-78.

39. Johansson M, Thurne A, Blomqvist A, et al. Management of acute cholecystitis in the laparoscopic era: results of a prospective, randomised clinical trial. J Gastrointest Surg 2003;7.642-5.

40. Kolla SB, Aggarwal S, Kumar A, et al. Early vs delayed laparoscopic cholecystectomy for acute cholecystitis. Surg Endosc 2004;18:13237.

41. Lai PBS, Kwong KH, Leung KL, et al. Randomized trial of early versus delayed laparoscopic cholecystectomy for acute cholecystitis. Br J Surg 1998;85:764-7.

42. Lo C-M, Liu C-L, Fan S-T, et al. Prospective randomized study of early versus delayed laparoscopic cholecystectomy for acute cholecystitis. Ann Surg 1998; 227:461-7.

43. Sockeel, MP. Massoure X, Chapellier K, Fixot R, Delpy T. Chirurgie précoce ou différée dans la cholécystite aiguë. La T2A va-t-elle mettre fin à la controverse ? Gastroentérologie Clinique et Biologique ; 2009 ; 33, . p. 223-3.

44. Sani R, James Didier L, Chaibou MS, et al. La Pratique de Laparoscopie chez L'enfant à L'Hoptal National de Niamey- Niger. J. Afr Chir Digest 2013; vol 13 (2) : 1515 - 1518.

45. Maresceaux J, Evrard S, Keller P, Miranda E, Mutter D, Haaften KV. La coeliovidéoscopie est-elle dangereuse en période d'initiation. Gastroentérol clin biol. $1992 ; 16: 875-878$.

46. Ludwig K, Lorenz D, Koeckerling F. Surgical strategies in the laparoscopic therapy of cholelithiasis and CBD stones. ANZ J Surg. 2002 ; 72(8) :547-52. [65]. Strasberg SM. Acute calculous cholecystitis. N Engl J Med. 2008. 358:2804-11. 